\title{
Transposition
}

Musique et Sciences Sociales

$4 \mid 2014$

Musique et conflits armés après 1945

\section{Annegret Fauser, Sounds of War. Music in the United States during World War II}

New York, Oxford, Oxford University Press, 2013, 384 p.

\section{Esteban Buch}

\section{OpenEdition}

\section{Journals}

Édition électronique

URL : http://journals.openedition.org/transposition/506

DOI : 10.4000/transposition.506

ISSN : 2110-6134

Éditeur

CRAL - Centre de recherche sur les arts et le langage

Référence électronique

Esteban Buch, «Annegret Fauser, Sounds of War. Music in the United States during World War II », Transposition [En ligne], 4 | 2014, mis en ligne le 15 juillet 2014, consulté le 22 septembre 2020. URL http://journals.openedition.org/transposition/506 ; DOI : https://doi.org/10.4000/transposition.506

Ce document a été généré automatiquement le 22 septembre 2020.

La revue Transposition est mise à disposition selon les termes de la Licence Creative Commons Attribution - Partage dans les Mêmes Conditions 4.0 International. 


\section{Annegret Fauser, Sounds of War. Music in the United States during World War II}

New York, Oxford, Oxford University Press, 2013, 384 p.

\section{Esteban Buch}

\section{RÉFÉRENCE}

Annegret Fauser, Sounds of War. Music in the United States during World War II, New York, Oxford, Oxford University Press, 2013, 384 p.

\section{NOTE DE L'ÉDITEUR}

Annagret Fauser parle des enjeux théoriques et méthodologiques de cet ouvrage dans l'entretien publié dans le troisième numéro de Transposition.

1 Sounds of War: l'ouvrage d'Annegret Fauser sur la musique aux États-Unis pendant la Seconde Guerre mondiale peut susciter deux malentendus, qu'il vaut mieux lever tout de suite. Premièrement, bien que l'auteure évoque son intérêt pour une " histoire acoustique » du conflit (p.9), ce qui suppose la prise en compte d'un vaste spectre de phénomènes sonores allant - disons, pour faire image - du bruit des armes au râle des mourants, en passant par toutes les voix, toutes les musiques et tous les silences, son travail ne s'inscrit pas dans le courant en plein essor des sound studies mais bien dans la perspective désormais plus classique d'une histoire culturelle de la musique. À propos, et deuxièmement, bien que le sous-titre mentionne la musique en général comme objet du volume, il y est question seulement de musique classique et d'opéra, tandis que sont à peine mentionnés le jazz, le swing et les autres genres populaires, dont l'importance fut pourtant prépondérante. La musique de film, dont le rôle politique sur le court terme et le rôle mémoriel sur le long terme sont à juste titre soulignés dans 
l'introduction, n'est pas davantage abordée. Autrement dit, les personnages de ce livre ne s'appellent pas Glenn Miller ou Duke Ellington, moins encore Marlene Dietrich, mais Aaron Copland, Roy Harris, Henry Cowell, Lily Pons, André Kostelanetz, Yehudi Menuhin, Kurt Weill ou John Cage, voire Eleanor Roosevelt, la First Lady qui en février 1942 déclara dans la revue Musical America que la musique était désormais «more valuable than ever » (p. 36).

2 Une fois ceci posé, empressons-nous de dire que Sounds of War est un livre formidable. D'ores et déjà il s'annonce comme une référence, non seulement pour les recherches sur la musique pendant la Seconde Guerre mondiale mais aussi pour les travaux sur toutes les musiques pendant toutes les guerres. Cela mérite d'être avancé en cette année du Centenaire de la Grande Guerre qui, commémoration oblige, verra paraître un certain nombre de travaux sur la musique pendant les années 1914-18. Sans préjuger de la qualité de ceux-ci, l'ouvrage de Fauser peut représenter un point de repère, autant pour des questions d'ordre méthodologique que par sa contribution empirique, et pas seulement pour l'histoire des États-Unis. En effet, alors qu'en France les études sur la vie musicale des années 1940-44 sont axées sur l'histoire sociale du régime de Vichy et de l'Occupation; alors qu'à propos de l'Allemagne, de l'Italie ou de l'Union Soviétique ce sont respectivement les régimes nazi, fasciste ou stalinien qui en tant que tels retiennent l'attention, la focale sur l'Amérique des années qui vont de Pearl Harbor à Hiroshima met au centre des débats - même si le terme n'est pas utilisé - la culture de guerre en tant que telle, voire la guerre elle-même.

3 Ce livre vient, comme on dit, combler une lacune historiographique, d'autant plus saillante que les années de la Seconde Guerre mondiale furent décisives pour l'image que les États-Unis allaient projeter d'eux-mêmes pendant toutes les décennies suivantes, sur le plan musical comme dans bien d'autres domaines. L'auteure commence d'ailleurs par rappeler qu'une mélodie tirée d'Appalachian Springs (1944) de Copland fut jouée en janvier 2009 lors de la première investiture présidentielle de Barack Obama, quatre ans après que ce dernier en personne avait tenu le rôle du récitant dans une performance de Lincoln Portrait (1942) du même Copland en commémoration des attentats du 11 septembre 2001. Et en réalité il s'agit moins d'une lacune que de plusieurs, car la diversité des thèmes traités aurait pu justifier à chaque fois le concours d'un chercheur différent, au lieu du tour de force que représente cette synthèse inaugurale faite sous une signature unique.

4 Le premier chapitre traite des individus, sous la forme d'un tour d'horizon des différentes formes d'engagement des musiciens. Le spectre est large qui va du cas du « soldat-musicien » Marc Blitzstein, enrôlé en août 1942 dans l'Armée de l'air et affecté jusqu'en mai 1945 à Londres, où il écrivit la Airborne Symphony en hommage à ses camarades d'armes, à celui d'un John Cage qui semble avoir tout fait pour éviter d'être appelé sous les drapeaux et qui composa en 1942 son grinçant et sceptique Credo in US, en passant par un jeune Yehudi Menuhin qui, exempté du service des armes en tant qu'unique soutien de sa famille, purgea sa mauvaise conscience en s'exposant inconsidérément au danger lors de ses nombreux concerts en soutien des troupes. Audelà de ce constat de diversité, le tableau qui se dégage n'est pas seulement celui d'une domination du sentiment patriotique, du reste largement partagé au sein de la nation, mais encore celui de l'anxiété particulière des musiciens classiques, désireux de prouver leur utilité pour l'effort de guerre et, réciproquement, surpris par l'importance que certains responsables attribuent à une "good music" parée de toutes sortes de 
vertus morales et civiques. Selon Fauser, ces années de guerre sont celles d'une politique culturelle volontariste visant à blanchir la musique classique et ses représentants contemporains de ses associations élitistes ou highbrow, ce qui se traduit non seulement par des efforts institutionnels conséquents en faveur d'une culture midbrow, mais aussi par une disposition des compositeurs à simplifier leur musique pour gagner en intelligibilité. En cela, pourrait-on ajouter, ils restent fidèles à une tradition remontant au commentaire de Beethoven sur La Bataille de Vittoria : «Il est certain que nous écrivons mieux quand nous écrivons pour le public et si nous écrivons vite ", que Roy Harris reformule à sa manière en parlant d'une musique "simple, simple, simple - something so direct that everybody could understand it » (cit. p. 253). L'héroïsme beethovénien va d'ailleurs trouver un avatar direct, toutes proportions gardées en matière de simplicité, dans l'ode to Napoleon Buonaparte d'Arnold Schoenberg (1942).

Les institutions sont le sujet du deuxième chapitre, qui décrit le rôle de la musique successivement au sein de l'Office of War Information créé par Roosevelt en juin 1942, comme dispositif de propagande visant l'ensemble de la population grâce à une intense programmation radiophonique et la mise en vedette d'antifascistes tels qu'Arturo Toscanini ; dans le cadre de la diplomatie culturelle menée par le Département d'État, et singulièrement par l'office of the Coordinator of Inter-American Affairs dirigé par Nelson Rockefeller, qui envoya Aaron Copland dans différents pays d'Amérique latine sensibles aux charmes du Troisième Reich; sous la forme d'initiatives à l'intention des troupes, comme la distribution de songbooks intitulés Hit Army Kit et d'enregistrements appelés V-Discs (avec V pour Victory), ainsi que l'organisation de concerts de solistes prestigieux ou d'orchestres en uniforme, le tout par les soins des U.S. Army Music Advisors, dont la liste est reconstituée aux pages 109 à 112 grâce aux archives du Subcommittee on Music du Joint Army and Navy Committee on Welfare and Recreation, conservées à la Library of Congress; enfin dans une attention soutenue pour la musicothérapie et ses vertus avérées ou supposées pour le traitement des soldats atteints de chocs post-traumatiques, ce que le jargon d'alors appelle leur reconditioning. À propos des musicothérapeutes, en majorité des femmes, Fauser suggère une dimension sexiste dans certaines critiques qui, cependant, pouvaient toujours faire fond sur la difficulté réelle de démontrer l'efficacité de ces méthodes - une remarque qui toutefois converge avec son observation du faible nombre de femmes impliquées dans l'ensemble des activités musicales, si l'on excepte le cas des interprètes.

6 Les quatre sections sur l'histoire des institutions sont des explorations, certes riches et sophistiquées, mais néanmoins partielles, de domaines qui, chacun, mériteraient un traitement détaillé. Ce deuxième chapitre est d'ailleurs un peu différent du reste du livre, qui prend pour objet les musiciens et leur production artistique. Le quatrième, par exemple, est consacré aux exilés, à commencer par Darius Milhaud, Bohuslav Martinů et Béla Bartók, dont les parcours et produits de ces années de guerre restent moins connus que ceux d'Arnold Schoenberg, Paul Hindemith ou Kurt Weill - l'époque américaine de ces figures ayant longtemps été une manière d'aborder la guerre ellemême, aux côtés des chapitres correspondants dans les biographies des compositeurs américains. À propos de Darius et Madeleine Milhaud, on retiendra du récit de Fauser les conditions éprouvantes de leur séjour californien et le refus du compositeur de prendre parti dans la querelle entre fidèles de Pétain et partisans de De Gaulle au sein de la communauté d'expatriés, au nom du mot d'ordre résumé dans une lettre de mai 1944 : «Suivre l'Amérique jusqu'à la délivrance de la France» (cit. p. 307n). Quant à la vaste production musicale qu'inaugure en août 1940 un Quatuor $n^{\circ} 10$ op. 218 rebaptisé 
opus americanum 1, c'est surtout l'opéra Bolivar (1943), sur un livret de Madeleine tiré d'un roman de Jules Supervielle, qui pour l'auteure concentre les enjeux de cette période - une œuvre que toutefois Milhaud, qui en ce libérateur latino-américain voyait surtout un exilé, échouera à faire représenter. On reconnaît dans cette négative l'état d'esprit d'institutions telles que le Met, encore moins favorable que d'habitude aux entreprises modernistes, fussent-elles modérées par des traits stylistiques associés à la tradition française, que le compositeur exploite surtout dans son ballet Jeux de printemps (1944) pour la compagnie de Martha Graham.

7 De ces exilés, c'est Kurt Weill, le compositeur «formerly German » devenu citoyen américain en 1943, qui semble avoir fait le plus de gestes en direction du nationalisme indigène, par exemple en collaborant avec le OWI. Fauser s'inscrit d'ailleurs en faux (p. 281n) par rapport aux spécialistes de Weill qui le dépeignent « thinking and acting like an American ", pour souligner au contraire ce que coûta à l'auteur de I'm a Stranger Here Myself (1943), aussi bien sur le plan psychologique que du point de vue de sa réputation, l'effort d'adaptation à sa nouvelle patrie. Cependant, ce sont bien sûr les œuvres de musiciens nés aux États-Unis qui se trouvent au cœur de l'analyse de la question de l'identité américaine, qui relie les troisième et cinquième chapitres et constitue sans doute la principale contribution du livre du point de vue proprement musicologique. Dans un premier temps, l'auteure explore les usages politiques du passé musical et notamment la prétention de faire de l'Amérique, face à une propagande nazie qui la décrit comme une nation sans culture, la nouvelle patrie de la culture universelle. Cela suppose surtout la revendication du répertoire classique, avec Beethoven en emblème de la démocratie, par exemple lors des concerts du Philharmonique de New York, et un Wagner joué au Met sans discontinuer malgré des exilés qui, tel le musicologue Paul Henry Lang, y entendent une «background music for Mein Kampf » (p. 172). Seul l'officier américain de Madame Butterfly semble avoir vraiment posé problème, au point que cette œuvre disparaît du répertoire jusqu'à la veille de l'Armistice. Au-delà des mentions rituelles du melting-pot et des efforts de certains musiciens noirs comme William Grant Still pour faire valoir un héritage culturel différencié, c'est dans l'intérêt renouvelé pour l'époque de la Révolution américaine, en la personne du compositeur William Billings, et pour un folklore rural, blanc, masculin et largement mythifié que s'exprime le plus clairement l'idéologie nationaliste. Tout comme dans le projet d'américanisation de l'opéra, traduit par exemple dans A Tree on the Plains (1942) de Paul Horgan et Ernst Bacon; dans Oklahoma! (1943) de Richard Rodgers et Oscar Hammerstein II, aux frontières avec la comédie musicale ; ou encore dans une Carmen Jones (1943) du même Hammerstein, dont l'héroïne travaille non pas dans une tabacalera mais dans une usine de parachutes.

8 Le dernier chapitre est consacré à ce que l'auteure appelle " the music with the worst reputation in twentieth-century historiograpy» (p.11), à savoir ces d'œuvres où le nationalisme prend la forme d'Americana, c'est-à-dire de clichés patriotiques. American Salute de Morton Gould (1943), la Sixième Symphonie " Gettysburg » de Roy Harris (1944) et les œuvres de Copland ou Blitzstein déjà citées en sont des exemples de choix, avec une préférence générique pour les Great American Symphonies, parfois brandies en réponse à la «war symphony" Leningrad de Chostakovitch. L'hommage à des figures historiques telles que Jefferson ou Lincoln, l'emploi de chœurs et de récitants, la citation de mélodies patriotiques, les sonorités militaires, les moments funèbres au sein de trajectoires expressives triomphalistes font partie des ressources courantes de ce répertoire, régulièrement accompagné de louanges emphatiques de leur dimension 
authentiquement américaine. Et il ne semble y avoir eu guère qu'un Roger Sessions pour signaler que cet usage politique de la musique, ce que l'auteure appelle le «populisme musical » américain, rapprochait dangereusement les États-Unis des États fascistes qu'ils combattaient. De fait c'est bien une ressemblance de style et de fonction qui ressort de la comparaison, certes sommaire, entre ce répertoire d'Americana et les œuvres conçues à la même époque dans les pays de l'Axe ou en URSS. Face à cela, et audelà du comportement de John Cage sur le plan civique, son Credo in US, qui justement met au travail ces mêmes clichés sous la forme d'une « satire » moderniste, est présenté non sans paradoxe comme " one of the more [sic] emblematic pieces of Americana emerging from World War II » (p. 270).

9 Bref, « music mattered », dit Annegret Fauser à la toute fin du livre (p. 271). De ce rôle de la musique pendant la guerre, Sounds of War présente un panorama convaincant et rigoureux, fondé à la fois sur un très solide travail d'archives, sur une maîtrise avérée de l'histoire de la musique américaine et européenne du vingtième siècle, enfin sur une bonne connaissance de l'historiographie générale sur les États-Unis et la Seconde Guerre mondiale. En revanche, et pour finir sur une note critique, on peut regretter l'absence de réflexion d'ensemble sur le rôle des artistes, des écrivains et des intellectuels pendant le conflit, ainsi que celle (mises à part deux ou trois références sur le cinéma) de bibliographie sur d'autres types de production culturelle. Ces deux éléments auraient peut-être permis non seulement de désenclaver la discussion sur le nationalisme musical, mais encore de mieux cerner l'enjeu réel que la musique a pu représenter pour l'effort de guerre en général. Il n'est guère étonnant que de nombreux musiciens désireux de mettre en valeur leur métier aient proclamé que la musique est une arme, ou que la First Lady ait loué sa «force for morale » dans une revue musicale. Il est moins sûr que la conviction que la musique est une arme ait été partagée par les généraux en charge des opérations militaires, au-delà du fait que certains d'entre eux étaient sans doute heureux d'en pourvoir leurs hommes pour renforcer leur moral et les aider à tuer l'ennui ou la peur. 